\title{
Conceptual Discovery of Educational Resources through Learning Objectives
}

\author{
Stuart A. Sutton and Diny Golder \\ University of Washington and JES \& Co. \\ sasutton@u.washington.edu, dinyg@jesandco.org
}

\begin{abstract}
This poster reports on current work with the NSF-funded Achievement Standards Network (ASN) to support discovery of educational resources in digital libraries using conceptual graphs of officially promulgated achievement standards statements. Conceptual graphs or knowledge maps of achievement standards reveal the macrostructure of the learning domain modeled by those standards and support higher-level understanding by teachers and students. The work builds on the conceptual framework of the AAAS knowledge maps by providing the means to flexibly define and deploy new relationship schemas to fit the disparate modeling needs of the nearly 740 learning standards documents in the ASN repository. Using an RDF-based, node-link representation of learning goals and the relationships among them, the ASN Knowledge Map Service will provide the framework to correlate educational resources to nodes in conceptual models in order to augment more conventional mechanisms of discovery and retrieval in digital libraries.
\end{abstract}

Keywords: digital libraries; content and curriculum standards; knowledge maps; conceptual discovery.

\section{Introduction}

UNESCO identifies the various stages or levels in pre-college or pre-university education as Level 0 (pre-primary education) through Level 3 (upper secondary).[1] As part of this framework, jurisdictional authorities in most nations have formally promulgated achievements standards that specify what students studying at these levels should know and be able to do as a result of their education. Some such standards are promulgated at the national level and others at the level of states, provinces and other governmental subdivisions. Some nations have a single controlling set of standards and others like the United States have as many as 51 complete sets at the level of the states and the District of Columbia and even more at the local level within states.

The names provided these formally promulgated standards vary substantially across nations with little rationale for the variety in nomenclature. Some nations call them curriculum standards, others simply call them content standards, and still others identify them as frameworks. The component parts of these controlling standards are also identified by means of a rich array of names-standards, benchmarks, assessments and indicators. The list of names goes on ad infinitum. For the purposes of our 
research, we call all standards of this class, achievement standards since they all relate broadly to learning goals and student achievement.

Even given this rich nomenclature for achievement standards, there is a fairly universal framing of their intent and content. All achievement standards more or less indicate the knowledge and skills, the ways of thinking, working, communicating, reasoning, and investigating expected of students studying at UNESCO education Level 0 through Level 3. Achievement standards also enumerate the most important and enduring ideas, concepts, issues, dilemmas, and knowledge considered essential to the domain of study that should be taught and learned in schools under the jurisdiction of the standard's promulgating authority.

So defined, achievement standards are ontological (and frequently political) in nature-modeling the learning expectations of a people in their children and youth. Achievement standards reveal the macrostructure of the domain they model and, therefore, provide an additional mechanism for digital library access to educational resources correlated to those standards. Thus, metadata describing lesson plans, learning objects and other educational resources useful in meeting specific learning objectives may be assigned the identifiers for the achievement standards in which those objectives are embodied. Such assignments support searching by teachers, parents, students, curriculum developers and school administrators for appropriate resources to meet jurisdictional needs. In the United States, these achievements standards are beginning to be used as assessment categories for student learning through standards-based report cards.

This poster reports on the preliminary research involving advanced uses of a national repository of U.S. achievement standards called the Achievement Standards Network (ASN) that supports both research in standards-based education and the correlation of educational resources to achievement standards for various purposes ranging from enhanced information retrieval through standards compliance in teaching and learning.[2] In this research, we build on the information modeling of our earlier ASN research by exploring the power inherent in the standards data and the implicit and explicit relationships they embody in revealing the macrostructure of standards domains with the goal of enhancing the use of the standards by teachers and students in domain comprehension, exploration and resource discovery.

The goal of the original ASN work was to develop a conceptual schema and a networked repository of machine-addressable achievement standards that would serve immediate needs for true-to-source representations of the standards while being fully amenable to the Semantic Web. Using the Dublin Core Abstract Model as the framework in the original work, two entities were defined - the standard document and the statement. Statements represent the component achievement assertions contained in the standards documents. The standards documents are atomized into their component statements and represented in RDF with each standards document and each statement being assigned a dereferencable URI. To date, the ASN contains over 740 current and historical state and national U.S. standards documents with initial forays underway into including standards from non-U.S. jurisdictions. These documents are atomized into over 350,000 individual achievement statements.

The ASN data model also defines a set of structural relationships between individual statements creating hierarchical taxon paths comprised of RDF-triples that reconstruct the inter-statement context of the standards document. The work reported here 
is exploring additional, non-structural paths through the standards data based on semantic relationships deemed useful in interpreting and using the standards. These additional paths take the form of knowledge maps. One of the goals of the research is to provide a means for creating the most useful maps and the definition of the new properties necessary to the generation of those maps. The new properties defined will be used by ASN in the refinement of its standards authoring tool to support creation of new knowledge maps by authors of standards in the ASN repository.

"Knowledge maps are node-link representations in which ideas are located in nodes and connected to other related ideas through a series of labeled links."[3] The final form of the knowledge map is a directed acyclic graph. In work pre-dating the ASN, the node-link representation of achievement standards data was used by the NSF-funded National Science Digital Library (NSDL) to create a navigable visual representation of an achievement standards knowledge map.[4] The NSDL work demonstrated the utility of such maps in supporting development of higher-order cognitive skills necessary to knowledge acquisition and more successful retrieval of educational resources when those resources are mapped to nodes in the knowledge map. $[5,6]$.

The NSDL knowledge map work was based on the learning goals in the Benchmarks for Science Literacy [7] as visualized in the Atlas for Science Literacy [8] and provides visual representations that emphasize the interconnectedness of science concepts and the connections between learning goals and digital resources in the NSDL. Working closely with the authors of the Atlas, the NSDL researchers defined the set of explicit semantic relationships set out in Table 1 to serve as map edges.[9] In sum, the NSDL researchers derived a single schema of properties to represent the relationships inherent in a single standard-Benchmarks for Science Literacy.

Table 1. Supported relationships in the NSDL Concept Space Interchange Protocol (CSIP)

\begin{tabular}{|l|l|}
\hline Prerequisite & Is similar to \\
\hline Post-requisite & References \\
\hline Contributes to achieving & Is associated with \\
\hline Contains & Is referenced by \\
\hline Is aligned to & Supports \\
\hline Is closely related to & Contributes to and relies upon \\
\hline Is part of & Needs or requires \\
\hline
\end{tabular}

While the research reported here builds on the NSDL knowledge map conceptualization, it does not assume a fixed set of explicit semantic relations. Instead, we are exploring the nature of the explicit knowledge map relationships across standards documents from ten U.S. states to determine: (1) whether there is a common set of relationships inherent in standards knowledge maps regardless of the varying characteristics of the standards modeled; and (2) whether providing the capability of a core set of relationships with the ability to extend that set at the time of knowledge map creation results in more useful, expressive maps.

In addition to the investigation of explicit knowledge map relations-i.e., those explicit relationships defined in the authoring tool's configuration for a specific map, we 
are also exploring whether the automatic visual mapping of the structural relationships inherent in the existing ASN data produces mapping results more or less as useful as the human-authored knowledge maps. The goal of this second thread of inquiry is to determine whether the return-on-investment for the knowledge map authoring by humans exceeds that of the automatically generated mappings based solely on inherent document structure. Using the NSDL relationship schema noted in Table 1, preliminary results indicate that the prerequisite, post-requisite, contributes to achieving, contains and is part might prove useful and amenable to automatic identification. We are also exploring whether a hybrid system that relies first on the automatically generated map using the standards inherent structural properties as a base for human mapping augmentation serves user cognitive needs for visualizations of the macrostructure of the standards.

\section{References}

1. United Nations Education, Scientific and Cultural Organization (UNESCO): International Standard Classification of Education (1997), http://www.uis.unesco.org/TEMPLATE/pdf/isced/ISCED_A.pdf

2. Sutton, S.A., Golder, D.: Achievement Standards Network (ASN): An Application Profile for Mapping K-12 Educational Resources to Achievement Standards. In: Proceedings of the International Conference on Dublin Core and Metadata Applications, pp. 69-79 (2008)

3. O'Donnell, A.M., Dansereau, D.F., Hall, R.H.: Knowledge Maps as Scaffolds for Cognitive Processing. Educational Psychology Review 14, 71-86 (2002)

4. NSDL Strand Maps, http: / / knowledgemaps .nsdl . org/

5. Butcher, K.R., Bhusham, S., Sumner, T.: Multimedia Displays for Conceptual Discovery: Information Seeking with Strand Maps. Multimedia Systems 11(30), 236-248 (2006)

6. Sumner, T., Ahmad, F., Bhushan, S., Gu, Q., Molina, F., Willard, S., Wright, M., Davis, L., Janée, G.: Linking Learning Goals and Educational Resources through Interactive Concept Map Visualizations. International Journal of Digital Libraries 5, 18-24 (2005)

7. American Association for the Advancement of Science: Benchmarks for Science Literacy. Project 2061. Oxford University Press, Oxford (1993)

8. American Association for the Advancement of Science, National Science Teachers Association: Atlas of Science Literacy. Project 2061. Washington, D.C (2001)

9. Schema - CSIP.xsd, http://knowledgemaps.nsdl.org/cms1-2/docs/cms/ cms1-2/CSIPSchema/csip_doc.jsp 